Results RCS-levels among highly exposed workers were five times higher than the OEL and ten times higher in the early 1970s compared with 2000. Workers exposed to RCS had an increased risk of mortality from respiratory diseases, SMR 1.75 (95\% CI 1.22-2.44). The risk was more pronounced in men, SMR 1.86 (1.22-2.70). Among women, mortality from diseases of the circulatory system was increased, although not statistically significant.

A non-significant increase in the incidence of AMI was also observed, with slightly higher point estimates for women than for men. No dose-response relationship was observed in any analysis. We observed eight cases of silicosis, and seven appeared with more than 30 years latency.

Conclusions The RCS-levels at the porcelain factory were well above the OEL in the 1970s. We found an increased mortality from respiratory diseases which can be attributed to the harmful effects of RCS on the lung.

\section{FACTORS ASSOCIATED WITH LONG-TERM SICKNESS ABSENCE DUE TO MENTAL AND BEHAVIOURAL DISORDERS}

J S Silva-Junior, Fischer. Public Health School, University of São Paulo, São Paulo, Brazil

10.1136/oemed-2013-101717.13

Objective To evaluate the factors associated with long term absences from work due to mental and behavioural disorders, including the interactions of two theoretical models of occupational stress.

Methods A case control study was conducted among workers seeking social security benefits in São Paulo, Brazil. They were absent from work more than fifteen days due to medical conditions, confirmed by social security medical doctors. Cases were workers diagnosed with mental and behavioural disorders. Controls were patients with clinical disability due to other medical problems. Both groups, 160 cases and 225 controls, were randomly selected in an agency of Brazilian Social Security Institution. They were interviewed and answered a comprehensive questionnaire about sociodemographic data, habits and lyfestyle, occupational information, perception of work conditions and clinical history. Multiple logistic regressions were performed to evaluate the hierarchical association of independent variables with the outcome.

Results Mental and behavioural disorders were associated with females (OR 2.48, 95\% CI 1.43- 4.30), self-reported skin colour as white (OR 2.16, 95\% CI 1.30-3.59), higher school education (OR 2.07, 95\% CI 1.15-3.71), high tobacco consumption (OR 5.10, 95\% CI 1.55-16.80), high alcohol intake (OR 8.91, 95\% CI 1.2662.90), employed by a public company (OR 3.33, 95\% CI 1.0110.97), exposure to violence at work (OR 4.23, 95\% CI 1.929.28), perception of high strain combined with low social support and effort-reward imbalance combined with high overcommitment at work (OR 5.70, 95\% CI 2.67-12.18), and more than two clinical disorders in the past year (OR 2.66, 95\% CI 1.59-4.43).

Conclusions There are variables in all hierarchical levels associated with long term sickness absence due to mental and behavioural disorders. Occupational stressors are strongly associated with these conditions. The public health policies must consider social security data to establish guidelines for health promotion in primary level, in order to minimise social and economic impacts.

\section{ASSOCIATIONS BETWEEN COMMUTING AND SOCIAL CAPITAL IN A CROSS-SECTIONAL POPULATION SURVEY IN SOUTHERN SWEDEN}

K M Mattisson, Kristina, Stroh, Håkansson. Lund University, Lund, Sweden

\subsection{6/oemed-2013-101717.14}

Objectives Commuting has been claimed to increase the material wealth. Through geographically expanding peoples labour markets, through increased commuting possibilities, more work opportunities occurs that will strengthen the economy. Although economy and flexibility to choose where to live and work will improve for the individual, several studies have shown that health become aggravated with increased commuting. One plausible explanation to the negative health is reduced social capital. Social capital is a resource emerging from social relations and the strength of those, on an individual and societal level. This resource can be used to solve problems of individual and collective character. Our primary hypothesis is that the longer the commuting time the less time for social participation and thereby a lower social capital.

Methods The study design is cross-sectional and the study material consists of two retrospective data sets collected through questionnaires in 2004 and 2008. The study population consist of respondents working more than $30 \mathrm{~h} /$ week, 18 to 65 years old and answering questions about commuting $(\mathrm{N}=21,088$ persons). Commuting is defined as transportation from the home to the work place, characterised by duration one way $(<30 \mathrm{~min}$, 30-60 min, >1 h) and mode (active, car, public). Social capital is defined as a combination of questions on social participation and generalised trust to other people. Relationships between social capital and the exposure variable will be investigated with log-binomial regression. Social participation and generalised trust to will also be investigated separately.

Results Has not yet been produced but a primary analysis done with logistic regression (as an indicator, instead of logbinomial regression) seems to show an association between social participation and increasing commuting time both with car and public, compared to active commuting. Low generalised trust seemed not to be strongly associated with commuting.

(Results will be presented on EPICOH 2013)

\section{REPRODUCTIVE HEALTH OUTCOME FROM CHEMICAL EXPOSURE IN A DRUG LABORATORY IN MALAYSIA}

C W Hoe. University of Malaya, Kuala Lumpur, Malaysia

\subsection{6/oemed-2013-101717.15}

Introduction Chemicals in the workplaces have been known to cause various health effects to the workers range from just irritation to causing cancers. A risk assessment was conducted in a hospital based dangerous drug monitoring laboratory in Kuala Lumpur, Malaysia.

Methods The method is a Qualitative Risk Assessment method using the combination techniques of Hazard Identification, Risk Assessment and Control (HIRAC) and the Job Safety Analysis (JSA). A questionnaire survey on the medical and health problem faced by the staff was also distributed during the survey.

Results The assessment found that four of the workers gave adverse reproductive health outcome; a 25 years old female worker having history of spontaneous abortion and was unable to conceive, a 51 years old female worker has two spontaneous 